Microwave Engineering

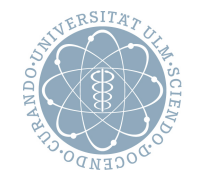

ulm university universität

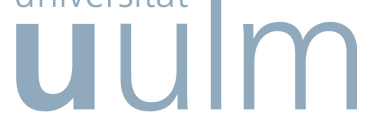

Thomas Galler, Tobias Chaloun, Kevin Kröhnert, Malte Schulz-Ruhtenberg, and Christian

Waldschmidt

(c) 2019 IEEE. Personal use of this material is permitted. Permission from IEEE must be obtained for all other uses, in any current or future media, including reprinting/republishing this material for advertising or promotional purposes, creating new collective works, for resale or redistribution to servers or lists, or reuse of any copyrighted component of this work in other works.

DOI: 10.23919/EuMC.2019.8910813 


\title{
Hermetically Sealed Glass Package for Highly Integrated MMICs
}

\author{
Thomas Galler $^{\# 1}$, Tobias Chaloun ${ }^{\#}$, Kevin Kröhnert*, Malte Schulz-Ruhtenberg ${ }^{+}$, and Christian Waldschmidt ${ }^{\#}$ \\ \#Institute of Microwave Engineering, Ulm University, 89081 Ulm, Germany \\ *Fraunhofer Institute IZM, 13355 Berlin, Germany \\ ${ }^{+}$LPKF, 30827 Garbsen, Germany \\ ${ }^{1}$ thomas.galler@uni-ulm.de
}

\begin{abstract}
A novel hermetically sealed RF packaging concept based on glass is presented. Using the laser induced deep etching (LIDE) technology enables the fabrication of glass vias without degrading the mechanical stability as micro-cracks are completely avoided. Furthermore, aspect ratios of up to 1:10 make this technology superior over conventional packaging solutions for the upper millimeter wave regime beyond $150 \mathrm{GHz}$. As an initial design demonstration, this paper shows a vertical RF-transition through the glass substrate using Through-Glass Vias (TGVs) with an aspect ratio larger than 1:6. The realized prototypes intended for highly efficient LO/VCO distribution within the glass package show excellent reproducibility with a maximum insertion loss of $0.4 \mathrm{~dB}$ up to $40 \mathrm{GHz}$. In addition, a very compact RF-interconnection from PCB to the glass package using solder balls is presented. The simulation of the RF-transition is in good agreement with the measured reflection and transition coefficient not exceeding $-15 \mathrm{~dB}$ and $-1.5 \mathrm{~dB}$ up to $35 \mathrm{GHz}$, respectively.

Keywords-Millimeter wave, transition, glass interposer, MMIC, PCB, TGV.
\end{abstract}

\section{INTRODUCTION}

The constantly growing operational frequency at MMICs is the main driver for highly integrated package solutions beyond $150 \mathrm{GHz}$. Due to the high frequency shrinking line width or spacing require an ultra compact housing concept, which is getting more difficult with conventional PCB materials [1]. In application scenarios with harsh environmental conditions like high temperature, humidity or high pressure, Teflon-based materials show diadvantages in mechanical stability and water resistance. Glass offers here a new technology for millimeter wave applications, in which inter-chip connections or interposer concepts can be realized for frequencies above

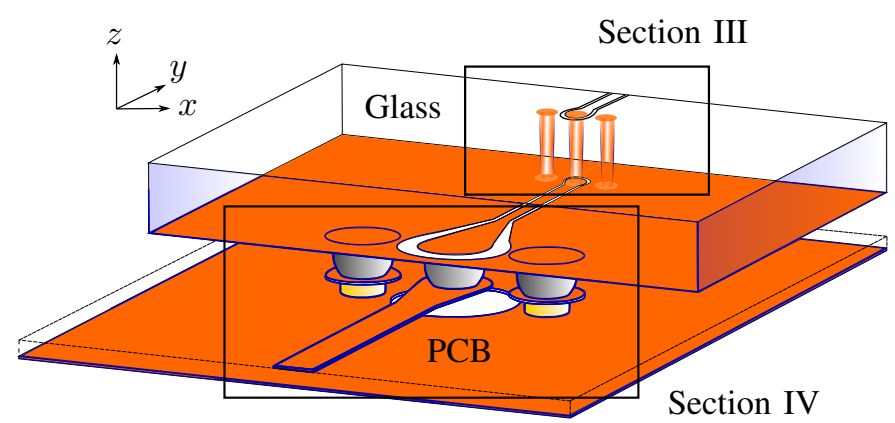

Fig. 1. Transition from microstrip line on PCB to coplanar line on the glass substrate using solder balls. A vertical transition through glass allows coplanar signal routing on both sides of the glass.

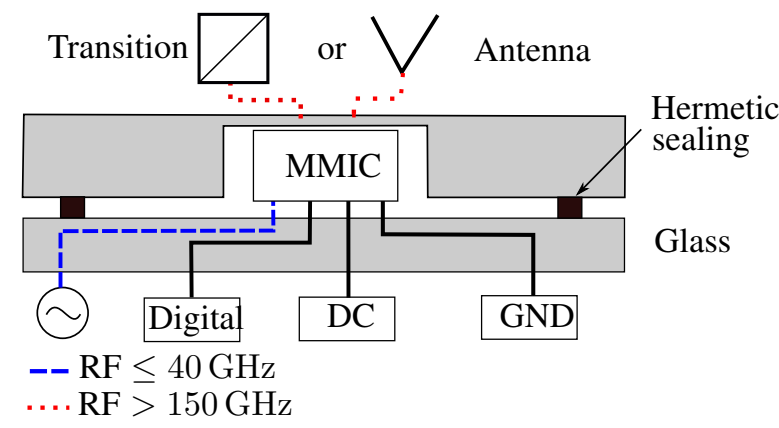

Fig. 2. Schematic of glass interposer concept.

$150 \mathrm{GHz}$. Various studies of Through-Glass Via (TGV) fabrication are presented as the key technology for advanced packaging techniques [2]. [3] presents an TGV implementation for interposers up to $20 \mathrm{GHz}$ comparing conventional fully filled TGVs with conformal metal plated ones. In [4], a glass wafer with a thickness of $400 \mu \mathrm{m}$ and TGVs with $85 \mu \mathrm{m}$ diameter results in an aspect ratio lower than 1:5 fabricated by a focused electrical discharging method (FEDM). In [5] single TGVs with $30 \mu \mathrm{m}$ width and $150 \mu \mathrm{m}$ depth were fabricated using a wet etching process.

Now this paper presents for the first time a complete RF interconnection design for a transition from microstrip line on the PCB to coplanar line on the top side of the glass. The TGVs are fabricated with a laser induced deep etching (LIDE) process from LPKF [6] which makes it possible to realize aspect ratios of 1:10 without cracks. Using this approach, a hermetically sealed glass package for MMICs is possible with very high wiring density as seen in Fig.2.

In this design a 3-wire line is implemented using TGVs with an aspect ratio larger than 1:6. Compared to the vertical transitions using a 3-wire line configuration realized in RO4003c [7], glass technology provides much less insertion loss. Due to the high surface hardness of glass much lower tolerances in the metalization accuracy are achieved which makes this technology suitable for high frequency circuit design beyond $150 \mathrm{GHz}$. Embedding the MMIC in the glass interposer structure creates a highly compact hermetically sealed glass package. The galvanic connection between PBC and glass is achieved by solder balls with a diameter of $400 \mu \mathrm{m}$. 


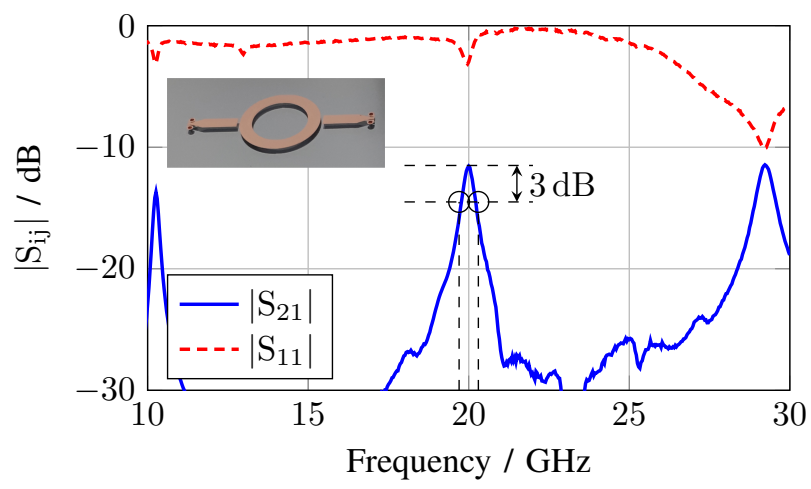

Fig. 3. S-parameter measurement of the ring resonator.

\section{Material Characterization}

Schott Borofloat B33 glass is a suitable material for RF applications, as its thermal expansion coefficient goes well with silicon in MMICs. For an appropriate design of the glass transition, precise information about the material parameters such as the effective permittivity $\varepsilon_{\mathrm{r} \text {,eff }}$ and loss tangent $\tan \delta$ are important. Therefore, a microstrip line ring resonator is designed for the frequency of $20 \mathrm{GHz}$. The diameter of the ring with multiples $n$ of the guided wavelength $\lambda_{G}$ allows the extraction of the effective permittivity [8]. Evaluating the $3-\mathrm{dB}$ bandwidth of the $n$th periodic resonance peak, the quality factor $Q$ can be determined from the loaded quality factor $Q_{L}$ at the measured frequencies $f_{R, n}^{(\text {meas }}$ [8]. As the resonator provides losses, an adaption of the measured resonance frequencies $f_{R, n}^{\text {(meas) }}$ to the nominal resonance frequencies $f_{R, n}^{(\text {real })}$ is done by the $Q$ factor as follows

$$
f_{R, n}^{(\text {real })}=f_{R, n}^{(\text {meas })}\left(1-\frac{1}{2 Q}\right)^{-1}
$$

Based on the length of the resonator

$$
l_{m}=\pi D_{m}=n \lambda_{R, n},
$$

the resonator ring diameter is dimensioned to $D=4.7 \mathrm{~mm}$ with $n=2$ for the $20 \mathrm{GHz}$ resonance frequency. Afterwards, the effective permittivity $\varepsilon_{\mathrm{r} \text {,eff }}$ and the loss tangent can then be calculated by

$$
\begin{aligned}
\varepsilon_{\mathrm{r}, \text { eff }}\left(f_{R, n}^{(\text {real })}\right) & =\left(\frac{c_{0} n}{\pi f_{R, n} D}\right)^{2}, \\
\tan \delta & =\frac{1}{Q_{L}\left(f_{R, n}^{(\text {real })}\right)} .
\end{aligned}
$$

Fig. 3 shows the S-parameters with $\left|\mathrm{S}_{21}\right|=-10.5 \mathrm{~dB}$ at the center frequency. Extracting $f_{R, 2}^{\text {(meas) }}=19.95 \mathrm{GHz}$, with the $3-\mathrm{dB}$ bandwidth $\Delta f_{2}=0.58 \mathrm{GHz}$, results in an $\varepsilon_{\mathrm{r}, \text { eff }}$ of 4.32 . The loss tangent is $\tan \delta=0.058$. Evaluating the different peaks results in $\varepsilon_{\mathrm{r} \text {,eff }}=4.41$ at $f_{R, 1}^{(\text {meas })}=10.33 \mathrm{GHz}$ and $\varepsilon_{\mathrm{r}, \text { eff }}=4.28$ at $f_{R, 3}^{\text {(meas) }}=29.16 \mathrm{GHz}$. These values are in good accordance to the ones extracted from 3D electromagnetic simulation software.

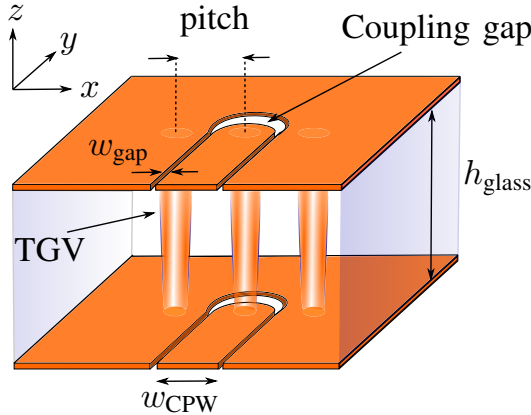

(a) Schematic cross section

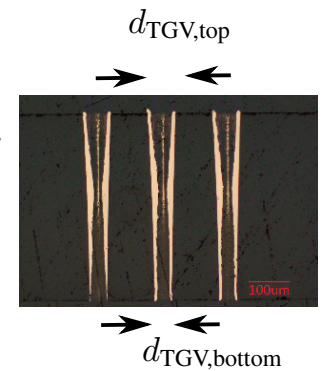

(b) Cut image of TGVs
Fig. 4. 3-wire line vertical RF transition using TGVs. The cross section shows the conical shape with inner metalization.

Table 1. Final dimensions of the simulated TGV transition.

\begin{tabular}{|c|c|c|c|c|c|}
\hline$w_{\text {gap }}$ & $w_{\text {CPW }}$ & pitch & $d_{\text {TGV,top }}$ & $d_{\text {TGV,bottom }}$ & $h_{\text {glass }}$ \\
\hline \hline $18 \mu \mathrm{m}$ & $100 \mu \mathrm{m}$ & $160 \mu \mathrm{m}$ & $80 \mu \mathrm{m}$ & $57 \mu \mathrm{m}$ & $490 \mu \mathrm{m}$ \\
\hline
\end{tabular}

\section{THROUGH-GLASS VIAS}

Based on the material characterization described in the previous section, a through-glass transition is developed. The design was optimized for a broadband transmission and a low insertion loss in the frequency range up to $40 \mathrm{GHz}$.

The LPKF Laser Induced Deep Etching (LIDE) [6] technology creates TGVs by locally activating glass with a laser pulse and a subsequent wet etching process. Thereby a reduction of production time is possible due to parallel processing of the glass wafer. Using this technology, the creation of micro-cracks can be avoided completely resulting in an increased integrability and a higher yield. High density packaging solutions are now feasible. For the vertical transition up to $40 \mathrm{GHz}$ a 3-wire line with TGVs is designed for a glass thickness of $h_{\text {glass }}=490 \mu \mathrm{m}$ and an upper TGV diameter of $80 \mu \mathrm{m}$ resulting in an aspect ratio of 1:6.

The proposed design is based on a coplanar transmission line structure on the top and bottom metalization of the glass, which is shown in Fig. 4a. This allows the signal and ground potentials to be on the same plane without the need of ground connections through the whole glass material. Moreover, the design is independent of the glass thickness as the field components are concentrated in the intervening gap. Strict specifications of height tolerances do not have to be met. The inductance and thus the impedance matching depend on the distance between the individual TGVs. Therefore, the dimensions were optimized by full wave simulations. The final parameters of the structure are listed in Tab 1.

To increase the bandwidth, the signal TGV has an expanded coplanar gap region (coupling gap) on the top and bottom metalization as shown in Fig 4. This improves the capacitive coupling and reduces local stray fields for a larger transmission bandwidth. The three TGVs are implemented based on a ground-signal-ground configuration as depicted in Fig. 4a.

The etching process leads to a conical shape of the TGVs along the vertical axis as depicted in the cross section shown 


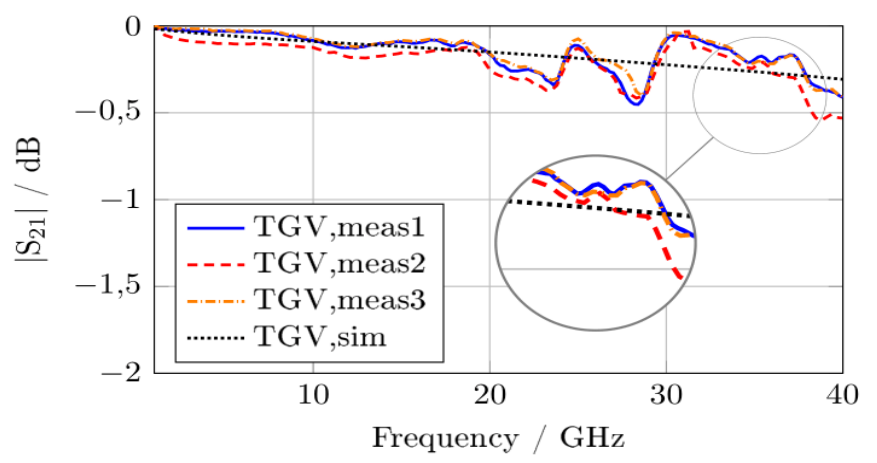

(a) Transmission coefficient

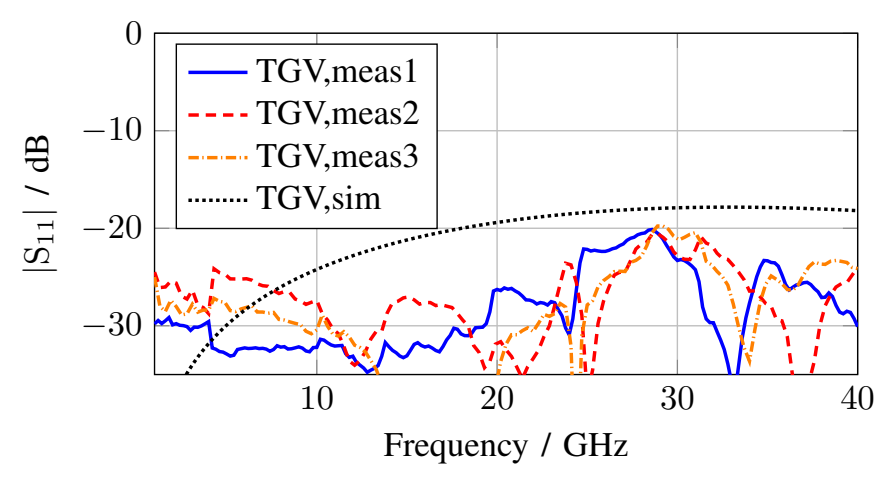

(b) Reflection coefficient

Fig. 5. Comparison of the measured and simulated results for the vertical TGV transition.

in Fig. 4b. After the etching process the inner metalization of the tubus and the coplanar structures on the glass surface are realized by a sputter process of titanium and copper from a single side resulting in a thickness of $4 \mu \mathrm{m}$ and an accuracy of $\pm 2 \mu \mathrm{m}$.

As simultaneous contacting by probe tips on the top and bottom glass metalization is impossible, a back-to-back measurement setup is used. This allows the characterization with both probe tips contacted on the top side. From the back-to-back configuration, shown in Fig. 6, it is possible to de-embed the performance of the single transition.

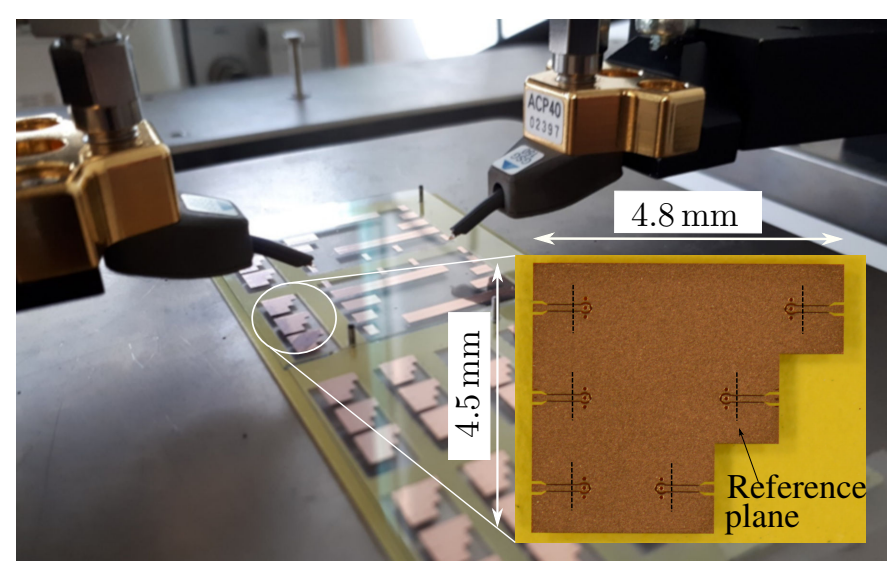

Fig. 6. Photograph of the measurement setup in back-to-back configuration.
The measured and simulated transmission coefficients $\left|\mathrm{S}_{21}\right|$ are compared in Fig. 5a. The insertion loss better than $0.4 \mathrm{~dB}$ shows a low pass characteristic over the whole examined frequency range of $40 \mathrm{GHz}$. The good accordance between the measured and simulated curves demonstrate the low fabrication tolerances in this glass technology. Excellent reproduceability can be seen in the small deviations of the measured curves. Fig. 5b shows the reflection coefficient. Compared to the simulation, the measurement curve for $S_{11}$ are below $-20 \mathrm{~dB}$. The losses of the coplanar line are de-embedded up to the reference plane.

\section{MSL-TO-CPW TRANSITION}

To connect the glass interposer structure with the microstrip line on the PCB a broadband transition based on a coplanar signal transmission is presented. Here a Ground-Signal-Ground (GSG) structure is used, which is aligned vertically on the PCB with solder ball contact pads, as depicted in Fig. 7. For PCB a Teflon-based RO 3003 material with a thickness of $127 \mu \mathrm{m}$ was chosen.

The ground metalization on the PCB has a hole aligned under the signal pad. This leads to a better guidance of the electromagnetic field along the solder balls, as the vertical stray fields are reduced. With the boundary conditions of a minimally remaining metal ring $(10 \mu \mathrm{m})$ around the vias, the diameter of the hole was optimized to $d_{\text {hole }}=870 \mu \mathrm{m}$. The capacitance and inductance are adjusted by the pitch of the solder balls as well as the vias in the PCB. The distance is set to $650 \mu \mathrm{m}$. Tab 2 summarizes the parameters for the investigated transition resulting from 3D-electromagnetic field simulations.

An alloy of Bismuth/Tin $(\mathrm{Bi} / \mathrm{Sn})$ solder balls with a diameter of $400 \mu \mathrm{m}$ connect the pads of the circuit board with the bottom metalization of the glass.

A further improvement to enhance the transmission bandwidth is achieved by slightly increasing the coupling gap $w_{\text {coup }}$ between the signal pad and the ground metalization on the glass substrate.

A reduction of $w_{\text {coup }}$ to the width of the coplanar line $w_{\text {gap }}$ is realized by a linear taper. The transition is optimized for

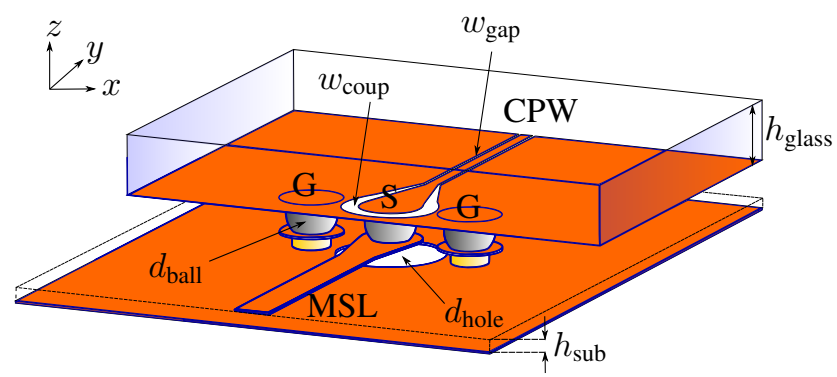

Fig. 7. Transition from the microstrip line on PCB to the coplanar waveguide on the glass substrate using solder balls.

Table 2. Final dimensions of the simulated Glass-PCB transition.

\begin{tabular}{|c|c|c|c|c|c|c|}
\hline$w_{\text {coup }}$ & $d_{\text {hole }}$ & pitch & $d_{\text {ball }}$ & $l_{\text {taper }}$ & $h_{\text {sub }}$ & $h_{\text {glass }}$ \\
\hline \hline $30 \mu \mathrm{m}$ & $870 \mu \mathrm{m}$ & $650 \mu \mathrm{m}$ & $400 \mu \mathrm{m}$ & $125 \mu \mathrm{m}$ & $127 \mu \mathrm{m}$ & $490 \mu \mathrm{m}$ \\
\hline
\end{tabular}




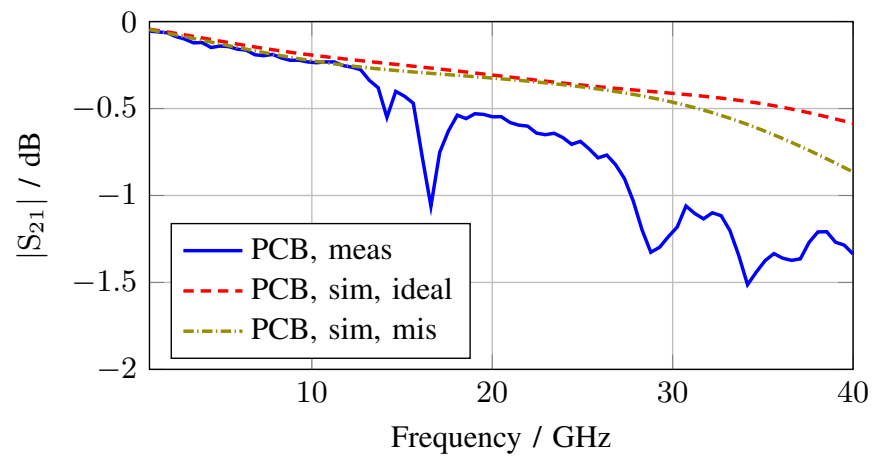

(a) Transmission coefficient

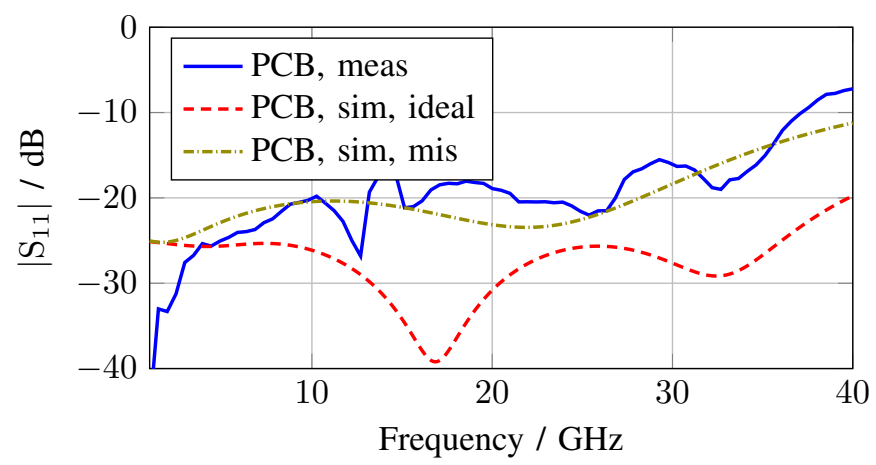

(b) Reflection coefficient

Fig. 8. Measured scattering parameters of the realized PCB to glass transition.

a low insertion loss in a frequency band up to $40 \mathrm{GHz}$. As the probes can only be contacted from the topside, again a back-to-back measurement setup in two different constellations is used in order to reduce deviations in the measurement. One constellation uses the contact of the probes on the PCB with glass in between (see Fig. 9a). The other one uses probe tips contacting on the glass with the PCB in the middle (see Fig. 9b).

Before the measurement results are presented, the fabrication chain is introduced. Again B33 glass is used and copper $(\mathrm{Cu})$ with a thickness of $4 \mu \mathrm{m}$ is galvanized into a patterned resist. Then, the resist and the seed layer is removed to pattern the bottom side of the glass. On top the solder balls are manually placed using solder resist and additional flux as an applicable standard process in industry. his guarantees stability under mechanical stress during the alignment of the PCB and the glass substrate. Afterwards a first reflow process is done to place the solder balls properly. The connection to the PCB material is achieved in a second reflow process at $140{ }^{\circ} \mathrm{C}$. The final distance between glass and PCB can be evaluated to $260 \mu \mathrm{m}$.

The measurement results of the PCB-to-glass transition are depicted in Fig. 8. Comparing the simulated and measured transmission coefficient the transition (see Fig. 8a), the insertion loss is less than $1.5 \mathrm{~dB}$ over the whole frequency range. Most probably the deterioration at higher frequencies is caused by misalignment of the solder balls caused by the

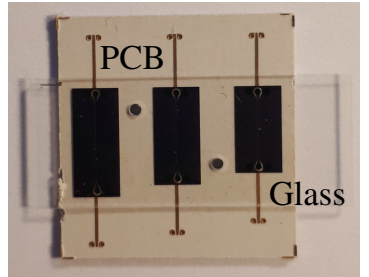

(a) Glass with probe pads on PCB

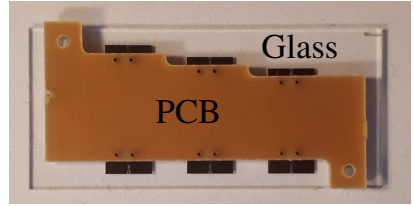

(b) PCB with probe pads on glass
Fig. 9. Photograph of fabricated PCB-to-glass transition.

manual placement. This can be seen in the comparison curve (PCB, sim,mis) created in a post simulation using the real pitch measured under the microscope. The skin effect is modeled by a reduction of the copper conductivity, which confirms the ohmic losses at higher frequencies. An input reflection coefficient of $\left|\mathrm{S}_{11}\right|<-16 \mathrm{~dB}$ can be measured up to $35 \mathrm{GHz}$, shown in Fig. 8b.

\section{CONClusion}

A first prototype of a RF hermetically sealed package implementation for PCB-to-MMIC interconnection is presented. For the transition from PCB to glass a maximum insertion loss of $1.5 \mathrm{~dB}$ was measured up to $40 \mathrm{GHz}$. The vertical transition through glass has a maximum measured insertion loss of $0.4 \mathrm{~dB}$, respectively. Glass offers here additional benefits especially in harsh environmental application scenarios compared to standard Teflon-based materials. Furthermore the LIDE glass technology is able to fabricate TGVs with an aspect ratio up to $1: 10$ which makes the concept applicable for designs beyond $150 \mathrm{GHz}$.

\section{ACKNOWLEDGMENT}

This work was funded by the VDI/VDE Innovation + Technik GmbH within the project GlaRA. - 16ES0692

\section{REFERENCES}

[1] K. Zoschke et al., "TSV based silicon interposer technology for wafer level fabrication of 3D SiP modules," in IEEE 61st Electronic Components and Technology Conference (ECTC), May 2011, pp. 836-843.

[2] K. Horiuchi et al., "Development of through glass vias (TGV) and through quartz vias (TQV) for advanced packaging," in International Conference on Electronics Packaging (ICEP), Apr. 2017, pp. 434-438.

[3] U. Shah et al., "Low-loss, high-linearity rf interposers enabled by through glass vias," IEEE Microwave and Wireless Components Letters, vol. 28, no. 11, pp. 960-962, Nov 2018.

[4] M. Tanaka et al., "Experimental study of through glass via effects on high frequency electrical characteristics," in Int. Conf. on Electronics Packaging and iMAPS All Asia Conf. (ICEP-IAAC), Apr. 2018, pp. 184-188.

[5] K. Xue et al., "Integrated process for high aspect ratio through glass vias," in 18th Int. Conf. on Electronic Packaging Technology, Aug. 2017, pp. 1451-1454.

[6] R. Ostholt et al., "High-throughput via formation in solid-core glass for IC substrates," MiNaPAD Conference, May 17-18, Grenoble, France.

[7] A. Stark and A. F. Jacob, "A broadband vertical transition for millimeter-wave applications," in 38th European Microwave Conference, Oct. 2008, pp. 476-479.

[8] R. Hoffmann and H. Howe, Handbook of Microwave Integrated Circuits, ser. Artech House microwave library. Artech House, 1987. 